\title{
Hypergraphia associated with a brain tumour of the right cerebral hemisphere
}

\author{
Toru Imamura, Atsushi Yamadori, Kenji Tsuburaya
}

Department of Neurology, Tohoku

Kohseinenkin

Hospital, Sendai,

Japan

T Imamura

K Tsuburaya

Neurology Service,

Hyogo Brain and

Heart Centre, Himeji,

Hyogo, Japan

A Yamadori

Correspondence to:

Dr Imamura, Department of

Neurology, Institute of Brain

Diseases, Tohoku University

School of Medicine, 1-1

Seiryo-machi, Aoba-ku,

Sendai 980, Japan.

Received 5 February 1991

and in revised form

17 April 1991.

Accepted 29 May 1991

Figure 1 Enhanced brain scan in 13th hospital week showing metastatic tumour as two adjoining masses with marked oedema in the right parietal region. Note intact left hemisphere and brain stem except minimal midline shift.

\begin{abstract}
Two different neurobehavioural abnormalities have been reported under the term hypergraphia. One has been described in temporal lobe epilepsies and the other in the acute stage of strokes of the right cerebral hemisphere. The latter type of hypergraphia in a patient with a metastatic brain tumour confined to the right hemisphere is reported. Such hypergraphia is a general right hemisphere sign that is not peculiar to strokes in the acute stage and the writing behaviour is inattentive.
\end{abstract}

Hypergraphia is now used for two different phenomena. Waxman and Geschwind originally defined this term as a tendency towards extensive and compulsive writing. ${ }^{1}$ They regarded it as one of the interictal behavioural changes in temporal lobe epilepsies. Recently Yamadori et al described another abnormal writing behaviour which was productive but inappropriate and occurred during the acute stage of right cerebral hemispheric strokes. ${ }^{2}$
They knew the previous usage of this term, but, nevertheless, they used the same term for this neurobehavioural abnormality and considered it to be a right hemisphere sign.

We report on a patient with hypergraphia who had a metastatic brain tumour of the right cerebral hemisphere.

\section{Case report}

An 80 year old previously healthy right handed man was admitted to hospital with a two week history of clumsiness of his left limbs. $\mathrm{He}$ was alert, well orientated, and scored $27 / 30$ on the Mini-Mental State Test (the cut off score for cognitive disturbances is 24 for the Japanese ${ }^{3}$ ). He had a moderate left hemiparesis and sensory disturbance. There was no anosognosia, left hemispatial neglect, or auditory or visual extinction, but he did show constructional apraxia when copying figures. Routine laboratory findings were all within normal ranges. A chest radiograph showed a coin lesion of his left lung, which was diagnosed as adenocarcinoma. Computed tomography of the brain showed a metastatic

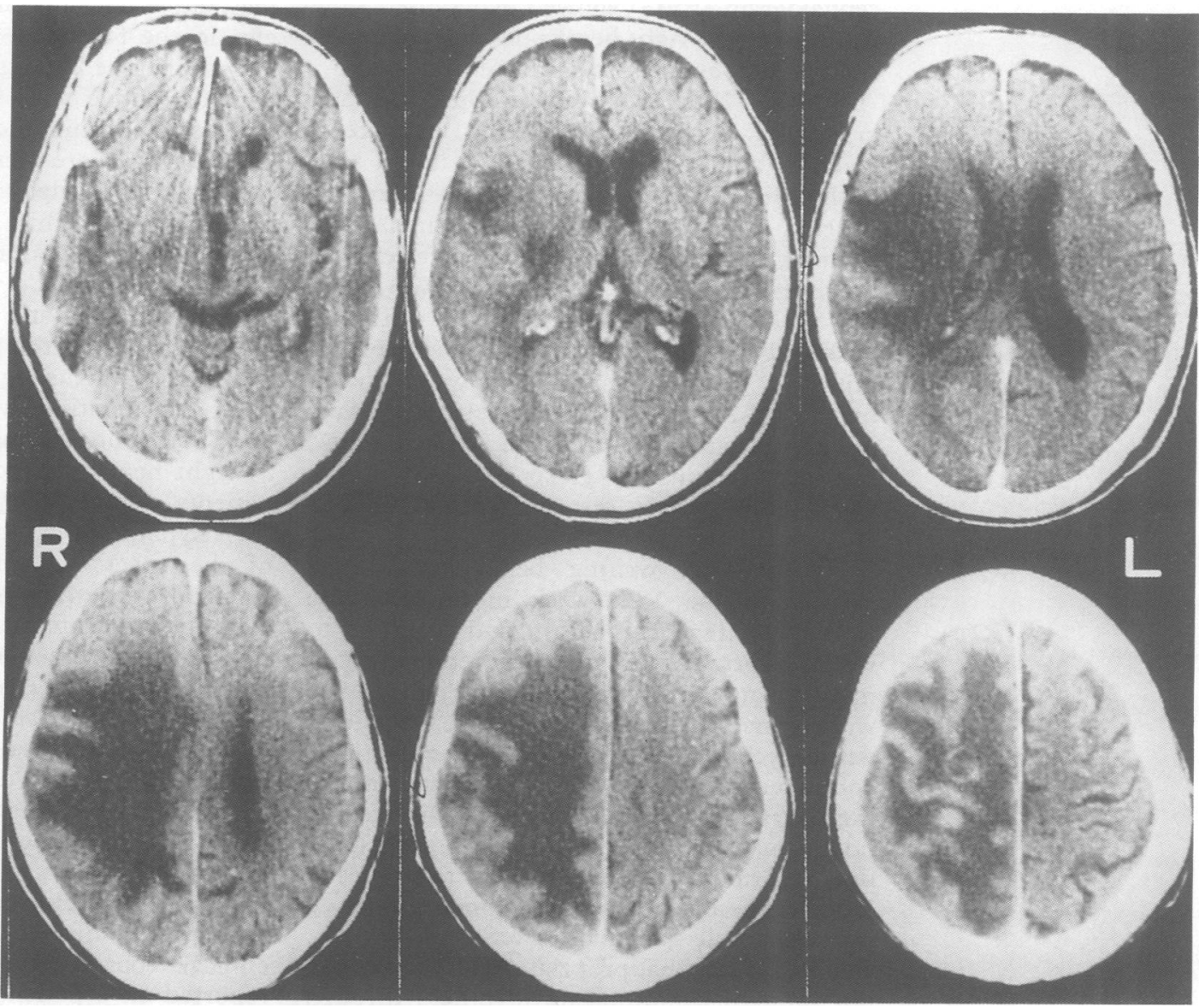




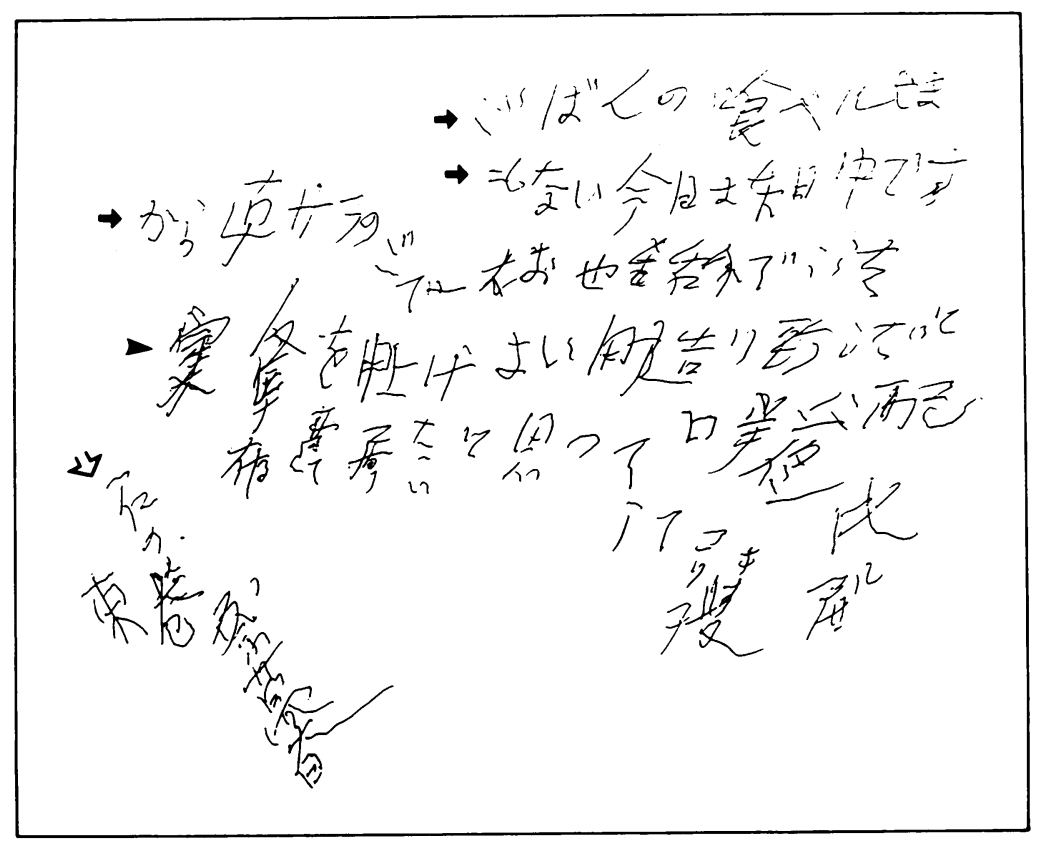

Figure 2 Example of patient's writing. Square indicates margin of the paper. Top, second, and third lines (arrows) are two sentences meaning "Too busy to eat meal" and "Please accept still more thanks because it is all through this month." On the fourth line (arrowhead) to the middle of the fifth line is another sentence, which translates as "I am going to report the actual condition for the campaign to construct better town." Name of a city near the home of patient is on the left lower corner (white arrow). Some letters on the lower half of this paper cannot be read because of severe spatial distortions.

tumour with marked oedema in the right parietal lobe. The weakness improved transiently with steroid treatment, but by the end of the sixth week in hospital he was hemiplegic. Occasional convulsive seizures limited to the left extremities were observed and were controlled with anticonvulsants. An electroencephalogram (EEG) showed generalised slow waves over the right hemisphere and sporadic sharp components in the right central area.

By the 10th week in hospital he had gradually become distracted, incoherent, agitated, and slightly delirious. A brain scan in the 13th week showed moderately increased oedema associated with the metastatic tumour, but the brain stem and left cerebral hemisphere remained intact (fig 1). We regarded the confusional state as identical with that observed in right cerebral hemispheric injuries. $^{4-6}$ The confusional state was followed by the appearance of left hemispatial neglect on line bisection tasks, when persisted, and ipsilateral instinctive grasp reaction ${ }^{7}$ of the right hand, which disappeared one month later.

His abormal writing behaviour became evident in the 15th week in hospital. He would spontaneously begin and continue to write. Every day he wrote the equivalent of more than 10 pieces of paper (fig 2). His writing manner was inattentive. He sometimes wrote over the written letters and sometimes continued writing on the table without noticing the margin of the paper. His writings had no mistakes as sentences but were spatially distorted and occasionally had extra strokes of letters. These sentences sometimes seemed to include simple questions or answers but their contents were inappropriate. He showed no intention to communicate to others through his writings. Once finished he paid no more attention to what he had written.

In the 18th week his confusional state subsided but his left hemispatial neglect and hypergraphia persisted. Repeated brain scans showed oedema and a mass effect essentially limited to the right hemisphere. Pneumonia developed in the 21 st week, and he died in the 25 th week of his illness.

\section{Discussion}

Serial computed tomograms of this patient showed oedema and the mass effect of a metastatic brain tumor confined to the right hemisphere throughout our observations. Right hemisphere signs appeared progressively as the tumour expanded. During this process altered writing behaviour was observed. First of all, we must evaluate several possible mechanisms of this behavioural abnormality.

Constructional disturbance can be a cause of spatial agraphia, ${ }^{8}$ to which we could attribute the twisted lines and extra strokes observed in his writings. Writing disturbances may also result from confusional states. ${ }^{9}$ But neither aetiology can fully explain the productive nature of this patient's writing behaviour.

This productive nature itself resembles the hypergraphia described by Waxman and Geschwind ${ }^{1}$ but associated characteristics do not. Waxman type hypergraphia is characterised by a compulsive tendency for writing, with a ritualistic and compulsive form, a detailed content, and the desire to keep what is written. ${ }^{1011}$ Our patient's writing, however, was characterised by extensive inattention: he was inattentive not only in general behaviour but also in the form and content of his writing. Yamadori type hypergraphia is semiautomatic and inattentive with spatial disarrangements and poor communicative value. ${ }^{212}$ These characteristics are identical with those of our patient. He was totally indifferent to his writing once finished. This attitude might be the additional behavioural feature of Yamadori type hypergraphia.

CTs, EEGs, and the other neuropsychological signs of this patient suggested diffuse dysfunction of the right cerebral hemisphere. This is compatible with the original report, which attributed Yamadori type hypergraphia to right hemisphere dysfunction. ${ }^{2}$ All of the patients originally reported had strokes and their hypergraphia subsided within four weeks of the onset. ${ }^{2}$ Okamura et al agreed that Yamadori type hypergraphia appeared in the acute or subacute stage of stroke and was a transient phenomenon. Our patient, on the other hand, suffered from a brain tumour. His hypergraphia appeared four months after the onset of neurological signs, continued more than six weeks, and did not show any sign of disappearance. These facts suggest that Yamadori type hypergraphia is not peculiar to the acute or subacute stage of strokes but can appear with various kinds of right cerebral hemispheric injuries at various stages of their clinical course. Recently Kanemoto et al reported on a patient with multiple sclerosis 
who manifested hypergraphic behaviour of Yamadori type for more than two years. ${ }^{13}$

1 Waxman SG, Geschwind N. Hypergraphia in temporal lobe epilepsy. Neurology 1974;24:629-36.

2 Yamadori A, Mori E, Tabuchi M, Kudo Y, Mitani Y. Hypergraphia: a right hemisphere syndrome. J Neurol Neurosurg Psychiatry 1986;49:1160-4.

3 Mori E, Mitani Y, Yamadori A. Usefulness of a Japanese version of the Mini-Mental State Test in neurological patients. Japanese Journal of Neuropsychology 1985;1: 82-90.

4 Mesulam MM, Waxman SG, Geschwind N, Sabin TD. Acute confusional states with right middle cerebral artery

5 Caplan LR, Kelly M, Kase CS, et al. Infarcts of the inferior division of the right middle cerebral artery: mirror image of Wernicke's aphasia. Neurology 1986;36:1015-20.

6 Mori E, Yamadori A. Acute confusional state and acute agitated delirium: occurrence after infarction in the right middle cerebral artery territory. Arch Neurol 1987; 44:1139-43.

7 Mori E, Yamadori A. Unilateral hemispheric injury and ipsilateral instinctive grasp reaction. Arch Neurol 1985; 42:485-8.

8 Heilman KM, Valenstein E, eds. Clinical neuropsychology. New York: Oxford University Press, 1985:87.

9 Chēdru F, Geschwind N. Writing disturbances in acute confusional states. Neuropsychologia 1972;10:343-53.

10 Roberts JKA, Robertson MM, Trimble MR. The lateralising significance of hypergraphia in temporal lobe epilepsy. $J$ Neurol Neurosurg Psychiatry 1982;45:131-8.

11 Joseph AB. A hypergraphic syndrome of automatic writing, affective disorder, and temporal lobe epilepsy in two patients. J Clin Psychiatry 1986;47:255-7.

12 Okamura T, Motomura N, Asaba H, et al. Hypergraphia in temporal lobe epilepsy: compared with stroke of the right cerebral hemisphere. Japanese Journal of Psychiatry and Neurology 1989;43:524-5.

13 Kanemoto K, Akamatsu T, Itagaki Y, Nishitani H. Early onset multiple sclerosis with hypergraphia and KlüverBucy syndrome: a case report. Neurological Medicine (Tokyo) 1990;32:301-3. 